\title{
Quels modèles de gestion des plantations agro-industrielles tropicales ? Réflexions à partir d'une étude de cas au Gabon
}

\author{
Jean-Philippe Tonneau ${ }^{1}$, Stéphane Guéneau ${ }^{2}$ \\ ${ }^{1}$ Géographe, Cirad, UMR9000 Tetis, Montpellier, France \\ 2 Socio-économiste, Cirad, UMR1110 Moisa, Montpellier, France
}

\author{
Mots-clés : \\ territoire ; \\ gouvernance ; \\ agro-industrie ; \\ développement \\ durable ; hévéa
}

\begin{abstract}
Résumé - Le fort développement de l'agro-industrialisation dans les zones tropicales fait craindre de graves conséquences sociales et environnementales. Des mécanismes de régulation permettent-ils d'atténuer ces conséquences ? À partir de l'étude d'une plantation industrielle d'hévéas au Gabon, les principaux enjeux sociaux et environnementaux sont d'abord détaillés. Nous présenterons les lacunes des modes de gestion mis en œuvre : responsabilité sociale et environnementale, politiques d'enclave et modèle paternaliste. Nous démontrerons que l'entreprise agro-industrielle ne peut répondre, seule, à l'importance des enjeux de développement que sa création suscite. Nous introduirons la notion de développement territorial, en précisant pour quelles raisons celle-ci permet d'appréhender et de gérer les transformations sociales et environnementales générées par les grands projets de plantation tropicale, malgré les difficultés de mise en œuvre que nous pressentons.
\end{abstract}

\begin{abstract}
Business models for managing tropical agro-industrial plantations: some considerations based on a study case in Gabon. The agro-industrial expansion in tropical areas raises serious concerns about its social and environmental impacts. Using an industrial rubber plantation in Gabon as a case study, our article first discusses the key social and environmental issues. We follow by presenting the limitations of the business models used: environmental and corporate social responsibility, enclave policies and paternalistic approach. We demonstrate that the private company model cannot meet alone the many sustainable development issues. These issues call for a better clarification of responsibilities among all stakeholders within a framework of planned territorial development, despite the structural difficulties that will probably stand in the way of its implementation.
\end{abstract}

L'agriculture industrielle modernisée à grande échelle s'est fortement développée sous les tropiques depuis les années 1990, en raison d'une demande mondiale soutenue de produits primaires (aliments, fibres, carburants...), demande relayée par des politiques nationales de soutien et d'attrait des investissements étrangers (Newton et al., 2013). Les conséquences sociales et environnementales négatives de la progression de l'agro-industrie sous les tropiques sont documentées et font l'objet de campagnes récurrentes des ONG depuis le milieu des années 2000 (Butler et Laurance, 2008; Gerber, 2011). Pour répondre à ces critiques et atténuer

Auteur correspondant : J.-P. Tonneau, jean-philippe.tonneau@cirad.fr 
les impacts de l'agro-industrie, les grandes entreprises ont mis en œuvre des stratégies de responsabilité sociale et environnementale (RSE), parfois en partenariat avec des ONG, souvent à travers des dispositifs de normalisation et de certification privés (Conroy, 2007 ; Vogel, 2008).

L'efficacité de ces stratégies de RSE appliquées aux secteurs agro-industriels a fait l'objet de plusieurs évaluations (Blackman et Rivera, 2010 ; McCarthy et Zen, 2010). Ces travaux portent sur un secteur d'activité, ou réalisent des comparaisons entre secteurs. Mais ils questionnent peu les effets des stratégies de RSE dans le contexte plus large de l'action publique au niveau d'un territoire. Quels sont les impacts de la plantation au-delà de ses limites et de ses environs proches? Comment les gérer? Où finit la responsabilité de l'entreprise ? Quel est le rôle de l'État?

Afin de répondre à ces questions, nous nous sommes appuyés sur l'étude du dispositif de RSE mis en œuvre par l'entreprise Olam-Rubber dans une plantation d'hévéas de la province du Woleu-Ntem, au nord du Gabon ${ }^{1}$. Olam-Rubber est une filiale du groupe industriel transnational Olam, l'un de ceux qui ont le plus fortement investi dans le développement des plantations agro-industrielles en Afrique. Olam-Rubber a obtenu un permis forestier associé (PFA $n \notin X 76 / 11$ ) sous la forme d'un bail emphytéotique de 50 ans renouvelable, concédé par l'État gabonais. Cette attribution s'inscrit dans le cadre du plan stratégique "Gabon émergent » promu par la présidence de la République (République gabonaise, 2012). Le Gabon a en effet affiché sa volonté de moderniser et diversifier son économie en s'appuyant sur un secteur agro-industriel moderne et performant, afin d'anticiper la diminution des revenus liés à la rente pétrolière et à l'exploitation forestière.

Dans un premier temps, nous décrivons le contexte local lors de la création de la plantation d'hévéa d'OlamRubber dans la province du Woleu-Ntem. Puis nous détaillons les enjeux sociaux et environnementaux auxquels est confrontée l'entreprise et nous analysons les stratégies qu'elle a développées pour y répondre. Ensuite, nous présenterons les deux grandes approches

\footnotetext{
1 Cet article est la valorisation d'une mission d'expertise d'analyse des impacts environnementaux et sociaux de la plantation d'Olam-Rubber dans la province du Woleu-Ntem au Gabon. Au cours de cette mission, nous avons réalisé des visites de terrain et une série d'entretiens avec une trentaine de personnes: villageois et chefs de village, responsables politiques locaux et nationaux, cadres de l'administration gabonaise, personnels et cadres d'Olam, membres d'ONG et de bureaux d'études. Une version de cet article a été présentée au congrès RIODD 2014 «Environnement et travail: quelles relations ? » (Bordeaux, 1-3 octobre 2014,

http: / / riodd2014.sciencesconf.org).
}

de gestion de ces enjeux qui ont été appliquées dans l'histoire par les entreprises : les politiques d'enclave et le modèle paternaliste territorial. Enfin, en conclusion, nous introduisons la notion de développement territorial, en indiquant pour quelles raisons celle-ci nous paraît appropriée pour gérer les transformations sociales et environnementales générées par les grands projets de plantations, malgré certaines difficultés de mise en œuvre que nous indiquons.

\section{La plantation industrielle d'hévéa d'Olam-Rubber dans le Woleu-Ntem : enjeux sociaux et environnementaux}

\section{Contexte de mise en œuvre de la plantation}

La situation de la province du Woleu-Ntem est particulière au Gabon, en raison de son homogénéité ethnique - elle est peuplée essentiellement par l'ethnie fang - et de sa densité démographique élevée en comparaison des autres régions gabonaises. L'ethnie fang s'étend aussi dans les régions limitrophes du Sud-Cameroun et de la Guinée équatoriale. Les flux d'échanges, tant humains que de marchandises, sont significatifs. Les populations du Woleu-Ntem sont fortement dépendantes des biens en provenance des pays voisins, notamment du fait du différentiel de prix conséquent entre les produits gabonais et étrangers (Loungou, 1999). La rente pétrolière et les salaires élevés contribuent à une faible productivité de l'économie gabonaise.

Pourtant, durant la période coloniale, l'agriculture familiale de rente (essentiellement café et cacao) a été relativement prospère. L'ethnie fang s'est fortement impliquée dans les programmes de développement de cette agriculture en améliorant les pratiques de production et en s'intégrant à l'économie monétaire (Didzambou, 2009). Comme toute agriculture familiale, elle était associée à des produits vivriers, essentiellement le manioc, l'arachide et la banane plantain. Les revenus de cette agriculture et l'alimentation des populations locales étaient, et sont toujours, complétés par la chasse, la pêche et la cueillette en forêt.

Le contexte agraire du Woleu-Ntem a fortement changé depuis les années 1970 en raison des effets de la rente pétrolière et de l'effondrement des cours mondiaux du café et du cacao. La production de cacao a chuté à partir de 1972, passant de 5000 à 3000 tonnes en cinq ans (Pourtier, 1980). En 2010, la production gabonaise de café et de cacao n'atteignait respectivement plus que 200 et 600 tonnes (N'Goulakia, 2014). Les cacaoyères qui subsistent à l'heure actuelle sont vieilles et peu entretenues par manque de main-d'œuvre et de débouchés. 
Dans les années 1980, le gouvernement a pris l'initiative de revitaliser l'économie rurale locale en misant sur le développement de l'hévéaculture. Cette culture avait été introduite dans le Woleu-Ntem durant la Seconde Guerre mondiale afin de répondre à la forte demande en caoutchouc des alliés. Les surfaces plantées qui atteignaient plus de 700 hectares ont été progressivement abandonnées après la fin de la guerre au profit d'autres cultures vivrières et de rente, essentiellement le cacao (Ovono Edzang, 2008). En 1981, les autorités gabonaises créent la société Hévégab. En 1985, une plantation industrielle d'hévéa est implantée à proximité de la ville de Bitam. Quelques années plus tard, des plantations villageoises sont créées (Enjalric et Ngoua Assoumou, 1998). Suite aux difficultés financières qu'elle a connues à la fin des années 1990, Hévégab a été privatisée et rachetée en 2004 par la société Siat-Gabon. Les plantations d'hévéas de Siat-Gabon s'étendent actuellement sur un peu plus de 7000 hectares.

Hormis ces plantations industrielles d'hévéas, l'agriculture est essentiellement vivrière (Magnagna Nguema, 2005). Au début des années 1990, les cultures itinérantes sur brûlis représentaient environ $28 \%$ des superficies de la province (Delpech et al., 1993). Les produits (toujours le manioc, la banane plantain et l'arachide) alimentent les marchés des capitales départementale, provinciale et nationale (Bitam, Oyem et Libreville). Certaines initiatives de diversification de l'agriculture et d'amélioration de la transformation des produits agricoles ont été menées sur financement d'ONG et du Fonds international de développement agricole. Elles restent très localisées et sont de faible taille.

Ces différentes politiques de développement rural conduites depuis l'indépendance ont connu des succès très limités. L'économie rurale du Woleu-Ntem a été fortement affectée par la croissance de l'économie pétrolière et par l'exode rural. Aux revenus historiques des productions de café et cacao se sont substitués les transferts de la migration vers les grandes villes, effet aussi de la rente pétrolière.

Ce contexte agraire a fortement façonné la situation sociale et environnementale de la province. La population jeune du Woleu-Ntem a progressivement émigré vers Libreville. Les villages sont peuplés essentiellement de jeunes enfants et de vieillards, ce que Pourtier décrivait déjà il y a plus de trente ans (Pourtier, 1980).

L'occupation agricole a repoussé les populations forestières nomades vers les zones boisées de l'est de la province. Les Pygmées Baka, population de chasseurscueilleurs, se concentrent autour de la ville de Minvoul. Minoritaires, ils entretiennent néanmoins des relations assez étroites avec les Fangs, avec qui ils communiquent en langue fang (Paulin, 2008).
L'agriculture vivrière et les plantations villageoises commerciales (cacao, café) développées en agroforesterie par ces populations rurales n'ont pas généré d'impacts environnementaux majeurs; cette situation peut être comparée à celle qui prévaut au sud du Cameroun voisin (Carrière, 1999). La forêt secondaire est en reconquête, à un stade de maturation relativement avancé sur les parcelles agricoles mises en jachère. Les Pygmées Baka dont l'activité agricole est récente mettent leurs connaissances traditionnelles de la forêt au service des systèmes agroforestiers qu'ils développent (Betsch, 2012).

\section{Un projet exemplaire du point de vue de la responsabilité sociale et environnementale?}

\section{Les enjeux}

Tout projet de plantation agro-industrielle génère des impacts environnementaux et sociaux parmi lesquels figurent, au premier plan, les changements d'usage des terres (Lambin et al., 2014). L'attribution de larges concessions à des entreprises multinationales conduit souvent à l'expulsion de petits agriculteurs familiaux aux droits fonciers traditionnels peu sécurisés, et souvent en situation d'insécurité alimentaire (Anseeuw et al., 2012; De Schutter, 2011). L'agro-industrialisation des tropiques a également de graves conséquences environnementales, par la conversion de forêts naturelles en plantations monoculturales (DeFries et al., 2010 ; del Carmen VeraDiaz et al., 2009 ; Gibbs et al., 2010). Les principaux effets environnementaux concernent les émissions de carbone relatives à la déforestation sur les zones de plantation (Carlson et al., 2013), la perte de biodiversité induite par la destruction des habitats naturels (Fitzherbert et al., 2008), l'érosion et les risques de contamination des eaux et des sols liés à l'utilisation de quantités importantes de produits chimiques (Hartemink, 2005).

La plantation d'Olam-Rubber n'échappe pas à ces enjeux environnementaux et sociaux. Ils ont été identifiés par l'étude d'impact environnemental et social (EIES) réalisée avant le démarrage du projet, disposition réglementaire obligatoire au Gabon (Enviropass, 2012). L'EIES indique que l'implantation du projet s'effectue sur des forêts secondaires, des vieilles jachères et des cultures, avec toutefois une présence de forêts moins perturbées jouxtant les ripisylves, dans une zone où le réseau hydrographique est relativement étendu. Cette situation s'explique par une activité agricole villageoise ancienne dans la région, mais en déclin depuis 40 ans. Compte tenu de la baisse progressive de l'activité agricole, la forêt perturbée tend à se reconstituer en forêt mature. 
Les espèces végétales présentes sur le site disposent d'un faible niveau d'endémisme et ne semblent pas devoir nécessiter des mesures de conservation particulières, sauf pour quelques-unes d'entre elles qui présentent un intérêt pour les populations villageoises (Enviropass, 2012). L'EIES souligne les risques d'impacts de la plantation sur la disparition de la macrofaune. L'effet des engins mécaniques sur les sols, les risques de ruissellement et de contamination des eaux de surfaces et souterraines, et la production de déchets industriels figurent parmi les autres enjeux environnementaux soulignés par l'EIES.

Les principaux enjeux sociaux concernent en premier lieu les conditions de travail des ouvriers de la plantation. L'empiétement sur des terres appartenant aux populations locales, ou sur lesquelles des droits d'usage leur sont reconnus, constitue un autre impact potentiel de la plantation. Enfin, la réduction des espaces utilisés par les populations pour leurs activités sylvestres (chasse, cueillette...) est également un aspect à prendre en considération (Enviropass, 2012).

\section{Les mesures}

Afin de prendre en charge ces différents enjeux, Olam-Rubber a mis en œuvre une série de mesures d'atténuation des impacts, conformément à la politique de RSE du groupe Olam. La première de ces mesures a consisté à réduire l'emprise de la concession de 36000 hectares à l'origine, à 28000 hectares, afin d'éviter l'empiétement sur des zones villageoises. Olam-Rubber a laissé une bande de $5 \mathrm{~km}$ autour des villages de la zone $\mathrm{du}$ projet, afin que les populations locales puissent poursuivre leurs activités forestières. Certains villageois ont cependant déclaré posséder des terres agricoles ou avoir des droits d'usage (chasse, cueillette, rites religieux) sur les forêts situées au-delà de cette bande préservée. Des consultations ont eu lieu entre l'entreprise et les populations villageoises afin de définir des compensations individuelles et collectives pour la perte de droits d'usage des forêts situées sur le lieu de la plantation.

Outre les compensations financières directes pour la perte de biens, des actions de compensation indirectes ont été inscrites dans des contrats sociaux. Elles se déclinent en trois grands types d'actions: la fourniture d'infrastructures de base (dispensaires, réhabilitation d'écoles et de logements, éclairage public, pompes hydrauliques, réhabilitation des routes, création de ponts, etc.) ; l'embauche prioritaire de main-d'œuvre locale à compétence égale ; l'appui à des activités génératrices de revenus (projets d'agriculture vivrière, par exemple).

L'atténuation des impacts sur la faune et la flore et la qualité des eaux a consisté à établir une bande de protection de 60 à 100 mètres autour des cours d'eaux sur l'ensemble du réseau hydrographique de la plantation. La collecte des déchets et leur stockage dans des lieux réservés à cet effet ont été organisés.

L'analyse de ces mesures nous a permis de conclure que la prise en charge des enjeux environnementaux par Olam-Rubber est proche des pratiques existantes en la matière pour d'autres projets de plantations pour lesquels il existe des standards volontaires de durabilité comme l'huile de palme par exemple, ce qui n'est pas le cas de l'hévéa.

Pour autant, la plupart des acteurs, y compris ceux chargés d'appliquer les mesures d'atténuation des impacts au sein d'Olam-Rubber, n'ont qu'une idée partielle des conséquences à venir, conséquences qui seront difficilement traitées au moyen d'une approche normative de type RSE.

\section{Des conséquences mal mesurées ?}

L'un des effets peu abordé par les différents experts concerne l'accroissement démographique rapide que risque de connaître la région. Dans des configurations africaines à peu près identiques, le total des migrants peut représenter jusqu'à sept fois le nombre d'employés de la plantation (Assembe-Mvondo et al., 2015). Les perspectives d'emplois dans la plantation d'Olam-Rubber étant de 7000 travailleurs, les flux de migration dans la région peuvent être estimés entre 35000 et 45000 personnes au cours des prochaines années, chiffres à comparer avec la population actuelle du chef-lieu du département, la ville de Bitam, qui compte environ 15000 habitants.

Comme dans d'autres régions, le salariat agricole est et sera assuré en grande partie par des travailleurs immigrés (Balac, 2001; Cramb et Curry, 2012). En sus de la main-d'œuvre, les flux d'immigration vont être constitués des familles des employés, de commerçants et d'artisans dont les activités accompagnent celles de la plantation, en fournissant des services complémentaires (alimentation, commerce de détail, etc.). Ces populations vont devoir être accueillies, logées, nourries. Cette évidence cache en fait de nombreux problèmes environnementaux et sociaux potentiels relatifs au foncier, à l'urbanisme et à la protection de la faune sauvage.

L'accès à l'alimentation peut être garanti à travers les salaires, mais une partie des populations allochtones cherchera vraisemblablement à développer une agriculture vivrière de subsistance. L'accès à la terre représentera alors un enjeu important. Or, les villageois gabonais sont très attachés à leurs droits d'usages ancestraux et sont peu enclins à les céder sans contreparties très élevées. L'accès au foncier risque d'être une source de tensions majeure, qui, en d'autres circonstances et d'autres lieux, a entraîné de graves conflits (Bossard, 2003). 
La croissance démographique et la réfection des routes sont susceptibles d'augmenter les opportunités de développement de la chasse commerciale dont les débouchés à Libreville restent très importants, malgré les interdictions et limitations réglementaires. La grande faune est très représentée dans le parc de Minkébé, situé à une trentaine de kilomètres du site d'exploitation, à l'est de Minvoul, alors même qu'il constitue déjà un terrain de chasse privilégié des braconniers. Entre 2004 et 2012, 11000 éléphants y auraient été tués (Maisels et al., 2014).

Face à ces enjeux cruciaux, la réflexion des dirigeants d'Olam, de l'État central, de l'administration déconcentrée et des collectivités territoriales nous semble insuffisante, en particulier parce qu'elle s'appuie sur des références historiques dépassées.

Le modèle colonial de la plantation a évité d'affronter ces enjeux en développant des politiques d'enclave. Le modèle du paternalisme territorial industriel en Europe au $\mathrm{XIX}^{\mathrm{e}}$ siècle, les a abordés en associant gestion de l'entreprise et gestion politique du territoire. Ces deux modèles nous semblent aujourd'hui impossibles à mettre en œuvre. Nous les présentons et analysons leurs inadéquations.

\section{L'enclave et le territoire}

\section{La plantation enclave}

Selon Brunet et al. (1992, p. 184), l'enclave « qualifie la situation d'une exploitation qui opère de façon autonome par rapport à son environnement immédiat, mais en relation suivie avec un partenaire extérieur ». L'enclave répond à des lois spécifiques, différentes de l'espace au sein duquel elle est insérée. L'espace concédé est considéré comme un stock de ressources dans lequel une entité extérieure au territoire puise, selon ses besoins (Veltz, 1993). Dunning et Lundan (2008) utilisent le terme de resource-seeking.

L'enclave induit des effets indirects faibles sur l'économie locale. Les investissements se limitent au secteur des transports, pour évacuer la matière première vers les pays consommateurs. Une fois les ressources épuisées, la firme se délocalise. L'enclave a souvent une durée fixe, généralement au moins équivalente à une génération humaine, négociée à travers une concession.

Aujourd'hui, l'enclave ne peut être affichée comme un objectif. Les législations, la pression de la société civile, locale et internationale, obligent les investisseurs étrangers à annoncer des liens avec des partenaires locaux pour éviter les conflits. Pourtant, l'enclavement existe toujours mais comme conséquence de conflits entre l'entreprise, les populations locales et parfois le gouvernement. L'enclavement est la sanction d'une impossibilité à gérer les relations complexes entre entreprises et territoires.

\section{L'enclave : une réponse à l'impossibilité de gérer des conflits structurels?}

Dans la plupart des cas recensés et documentés (Gerber, 2011), les conflits s'installent de manière progressive. Au départ, les populations locales sont généralement opposées à l'installation des plantations. Les compensations offertes par l'entreprise aux villageois pour la perte de leurs droits permettent d'atténuer temporairement les ressentiments. Au fil du temps et de la montée des coûts, les investisseurs se replient sur des logiques économiques alors que les populations sont plus exigeantes au fur et à mesure qu'elles constatent les impacts sur leur vie quotidienne. La satisfaction diminue et les conflits augmentent (Magrin et van Vliet, 2006).

Sans préjuger de l'avenir et sans douter de la volonté des dirigeants d'Olam-Rubber de contribuer au développement de la région, ce scénario de détérioration des relations entre l'entreprise et son environnement n'est-il pas inéluctable? L'histoire de l'implantation d'OlamRubber au Woleu-Ntem tend à démontrer une similitude des trajectoires.

Pour désamorcer les conflits initiaux avec les populations riveraines de la plantation, Olam-Rubber s'est engagé dans un programme social ambitieux : création ou réhabilitation de bâtiments publics (écoles, dispensaires, etc.), éclairage public, pompes, réfection des routes, etc. Bien que toutes ces actions soient inscrites dans des contrats sociaux signés par les représentants des villageois, les demandes et attentes des populations ne se limitent pas aux engagements contractuels. Des jalousies et des conflits entre villages sur la localisation des investissements apparaissent (tous veulent tout, chez eux). La question de la définition des cartes scolaires et sanitaires se pose avec acuité.

Les attentes s'orientent aussi de plus en plus vers des actions purement privées comme le défrichage des parcelles ou l'aide à la construction de logements. Les demandes des autorités locales relèvent de la même ambiguïté : il est attendu de l'entreprise qu'elle contribue fortement aux questions d'urbanisme, de sécurité, de santé et d'éducation.

Olam-Rubber est invitée à gérer des situations complexes, certes de sa responsabilité, mais d'une responsabilité indirecte, dans un processus de compensation infinie. L'expression qu'a utilisée un responsable villageois lors d'un entretien est significative : "Olam doit être un père pour nous ». 


\section{La tentation - impossible - du « paternalisme territorial »}

Le paternalisme est défini comme le caractère familial des relations entre employeurs et employés. Le patron assume l'autorité et les devoirs d'un père à l'égard de ses « enfants salariés ». Il est responsable de leur bien-être en contrepartie de quoi ils lui doivent respect et obéissance (Brand et Durousset, 1991).

Certaines des mesures appliquées par Olam-Rubber dans le cadre de la RSE s'inscrivent dans cette logique, en particulier en ce qui concerne la fourniture de logements et autres services (économats, poste médical, etc.). Des systèmes de protection sociale sont aussi mis en œuvre.

Paradoxalement, ces mesures contribuent aux tensions et à terme à l'enclavement. Les populations non concernées par ces avantages souhaitent en bénéficier. L'entreprise ne peut les satisfaire sauf à mettre en péril sa pérennité économique. Se pose ici la question de la portée de la RSE. Où s'arrête-elle ? Doit-elle s'appliquer aux effets indirects ? L'entreprise peut-elle assumer des responsabilités territoriales?

Le paternalisme industriel s'est parfois impliqué dans la gestion du territoire politique (Edelblutte, 2010). Les cas de Michelin à Clermont-Ferrand, de Schneider au Creusot ou des Houillères à Anzin sont significatifs d'un territoire construit pour et autour de l'usine. L'entreprise et le patron font œuvre d'aménagement du territoire. Ce modèle que nous pourrions qualifier de "paternalisme territorial » est probablement celui imaginé par nombre des acteurs impliqués peu ou prou dans le développement des plantations agro-industrielles. Mais de nombreuses différences nous semblent interdire la réplication de ce modèle.

La première est liée aux doctrines qui pilotent l'économie. Les entreprises de l'ère du paternalisme industriel se sont construites dans le cadre de politiques nationales pour l'édification d'industries stratégiques nationales (les mines, la sidérurgie, l'automobile, les armements, etc.) en des temps de confrontation et de préparation à la guerre. La préférence nationale limitait l'exigence de compétitivité. Aujourd'hui, la globalisation s'est imposée, les firmes sont mondiales, leur management est d'abord financier et impose la compétitivité.

De façon plus déterminante, le caractère mondialisé des entreprises empêche toute implication politique locale. Dans le modèle du paternalisme industriel territorial, les industriels ou leurs représentants exerçaient tous un rôle politique, local et national. Ils étaient maires, conseillers généraux, députés ou sénateurs. Grâce aux fonctions de député et de sénateur, les industriels pouvaient agir au niveau national et obtenir les appuis nécessaires aux politiques d'infrastructures. Les patrons ont ainsi pu mener des gestions intégrées, rendues possibles par une double légitimité : économique et politique.

Aujourd'hui, ces légitimités sont de plus en plus contestées. Les entreprises agro-industrielles sont toujours soumises au double contrôle des ONG (critiques de politiques considérées comme d'accaparement et $\mathrm{d}$ 'exploitation de ressources) et des politiques, désireux de profiter de la production de richesses.

\section{Conclusion : l'alternative du développement territorial}

Les entreprises ne peuvent gérer les impacts environnementaux et sociaux des plantations agro-industrielles sans aborder leurs effets indirects au niveau plus large $\mathrm{du}$ territoire. La firme mondialisée ne peut répondre, seule, aux enjeux de développement que son action suscite. Elle n'en a ni les moyens économiques ni la légitimité politique. Ce qui était anciennement possible dans les fronts pionniers, des infrastructures exclusivement focalisées sur l'exploitation des ressources, n'est plus acceptable dans des zones aujourd'hui plus peuplées. Les investissements sont contraints, non seulement par la réglementation (obligation d'études d'impacts), mais surtout par la pression sociale, d'apporter des garanties quant à leur prise en charge des impacts environnementaux et sociaux de leurs activités.

Dans l'idéal, la contribution de l'entreprise à l'économie régionale passe par les achats qu'elle effectue, par les sous-traitances qu'elle contracte, par les salaires qu'elle distribue et surtout par les impôts qu'elle paie (Bloch et Owusu, 2012 ; Magrin et van Vliet, 2006). Dans l'idéal, l'État investit en infrastructures en les gageant sur les revenus des impôts des entreprises agro-industrielles et des activités commerciales et industrielles qui en découleront.

Mais l'État est souvent faible. Cette faiblesse est parfois perçue par les firmes comme une facilité permettant des investissements à bas coûts grâce aux exonérations fiscales, pierre angulaire de nombre d'accords d'investissements. Mais en l'absence de mesures fiscales robustes, l'État pourra difficilement jouer son rôle d'aménageur et garantir les infrastructures nécessaires. Et compte tenu des risques de crises sociales et environnementales qui peuvent paralyser les activités économiques, l'État est confronté à des difficultés dans sa fonction de régulation.

Dans cette perspective, il importe de mieux définir les responsabilités des parties prenantes. Dans la continuité des travaux de Hirschman (1958), l'enjeu est bien d'améliorer les liens économiques et spatiaux entre entreprise et territoire à travers des politiques publiques (sectorielle, aménagement du territoire, développement local). Ce sont en fait les relations entre firme, État et 
population locale qu'il convient de repenser pour dépasser les contradictions actuelles et inventer un nouveau mode de gestion.

Inventer. Le mot est juste car ni les solutions, ni même les expériences convaincantes n'existent. Un autre modèle, celui du développement territorial nous paraît intéressant pour atteindre un objectif de durabilité de la plantation. Le concept du développement territorial est polysémique (Angeon et al., 2007). Il désigne à la fois : un objectif, l'amélioration du mieux-être collectif d'une population dans un espace donné ; le processus de transformation des structures économiques, sociales, culturelles, environnementales pour atteindre cet objectif ; la démarche, les outils et les méthodes pour promouvoir ce processus dans un "territoire en train de se faire" (Latour, 1999).

Dans de nombreux pays du Sud, du fait des difficultés des États, le développement territorial est apparu comme une voie permettant, soit de consolider des dynamiques endogènes de développement local, soit de les favoriser, voire les susciter. Mais est-il réaliste de promouvoir de telles démarches quand la décentralisation n'a pas été achevée ou que les collectivités territoriales manquent souvent de moyens financiers, humains et institutionnels ? Est-il réaliste de défendre la voie du développement territorial quand la décentralisation se traduit par de nouvelles relations de pouvoirs très éloignées des idéaux démocratiques?

Les rares évaluations montrent que le développement territorial, en Europe comme aux Suds, vaut surtout par l'accumulation de capitaux humain et social qu'il suscite (Koop et al., 2010). Les succès sont souvent dépendants de la liberté administrative et financière donnée à des expériences pilotes et à une logique de projet. En termes d'activités économiques et de création de richesse, le développement territorial reste confiné à des opérations à fort investissement humain, à des activités innovantes ou alternatives (comme l'agroécologie). La difficulté à mobiliser du capital financier dans ces opérations innovantes ou alternatives, donc à haut risque, est patente. Les facteurs économiques classiques (dotations en facteurs, potentiel de marché, économies d'agglomération...) restent les déterminants essentiels de la situation économique, et donc de l'état de développement.

Si la piste du développement territorial semble intéressante, elle reste néanmoins confrontée à des difficultés structurelles qu'il convient de ne pas minimiser. L'absence de réflexion structurée et avancée sur le développement territorial au niveau local et les compétences limitées des administrations nationales et locales en matière de développement territorial sont révélatrices de l'ampleur de la tâche.

\section{Remerciements}

Cet article est le résultat d'un travail de terrain entrepris en partenariat avec la société Olam-Rubber Gabon. Nous tenons à remercier Messieurs Gagan Gupta, Christopher Stewart et Olivier Desmets d'Olam-Rubber Gabon, ainsi que toutes les personnes rencontrées lors de notre mission, pour leur appui et leur disponibilité.

\section{Références}

Angeon V., Moquay P., Lardon S., Loudiyi S., Poss Y., Pivot J.M., Caron A., 2007. Le développement territorial : principes et méthodes, in Lardon S., Moquay P., Poss Y. (Eds), Développement territorial et diagnostic prospectif. Réflexions autour du viaduc de Millau, La Tour-d'Aigues, Éditions de l'Aube, 27-59.

Anseeuw W., Alden Wily L., Cotula L., Taylor M., 2012. Land rights and the rush for land. Findings of the global commercial pressures on land research project, Rome, International Land Coalition.

Assembe-Mvondo S., Cerutti P.O., Putzel L., Eba'a Atyi R., 2015. What happens when corporate ownership shifts to China? A case study on rubber production in Cameroon, European Journal of Development Research, 13, doi: 10.1057/ ejdr.2015.13.

Balac R., 2001. Dynamiques migratoires et économie de plantations, in Tapinos G.-P., Hugon P., Vimard P. (Eds), La Côte d'Ivoire à l'aube du XXI siècle. Défis démographiques et développement durable, Paris, Karthala, 195-231.

Betsch J.M., 2012. L'agriculture itinérante sur brûlis : quelques pratiques particulières des Pygmées du Gabon et les raisons qu'ils en donnent, Journal des africanistes, 82, 193205.

Blackman A., Rivera J., 2010. The evidence base for environmental and socioeconomic impacts of "sustainable" certification, RFF Discussion Paper, RFF-DP 10-17, http:/ / www.rff.org/files/sharepoint/WorkImages / Download/RFF-DP-10-17.pdf.

Bloch R., Owusu G., 2012. Linkages in Ghana's gold mining industry: challenging the enclave thesis, Resources Policy, 37, 434-442.

Bossard L., 2003. Peuplement et migration en Afrique de 1'Ouest: une crise régionale en Côte d'Ivoire, Afrique contemporaine, 206, 151-165.

Brand D., Durousset M., 1991. Dictionnaire thématique histoire géographie, Paris, Sirey.

Brunet R., Ferras R., Théry H., 1992. Les mots de la géographie: dictionnaire critique, Montpellier/Paris, Reclus/La Documentation française.

Butler R.A., Laurance W.F., 2008. New strategies for conserving tropical forests, Trends in Ecology \& Evolution, 23, 469-472.

Carlson K.M., Curran L.M., Asner G.P., Pittman A.M., Trigg S.N., Adeney J.M., 2013. Carbon emissions from forest conversion by Kalimantan oil palm plantations, Nature Climate Change, 3, 283-287. 
Carrière S., 1999. Les orphelins de la forêt. Influence de l'agriculture itinérante sur brûlis des Ntumu et des pratiques agricoles associées sur la dynamique forestière du sud Cameroun. Thèse de doctorat, Montpellier, Université de Montpellier.

Conroy M.E., 2007. Branded! How the 'certification revolution' is transforming global corporations, New Society Publishers.

Cramb R., Curry G.N., 2012. Oil palm and rural livelihoods in the Asia-Pacific region: an overview, Asia Pacific Viewpoint, 53, 223-239.

De Schutter O., 2011. How not to think of land-grabbing: three critiques of large-scale investments in farmland, The Journal of Peasant Studies, 38, 249-279.

DeFries R.S., Rudel T., Uriarte M., Hansen M., 2010. Deforestation driven by urban population growth and agricultural trade in the twenty-first century, Nature Geoscience, 3, 178-181.

del Carmen Vera-Diaz M., Kaufmann R.K., Nepstad D.C., 2009. The environmental impacts of soybean expansion and infrastructure development in Brazil's Amazon Basin, Global Development and Environment Institute Working Paper, 09-05, Tufts University.

Delpech B., Flitner M., Hulshof M., Toueilles J., Twagiramungu F., 1993. Food production and trade dynamics in the N'Tem District, northern Gabon, Documents de travail ICRA, La Haye.

Didzambou R., 2009. La dynamique Fang dans la «mise en valeur » du Gabon pendant la période coloniale (18501960), Outre-mers, 96, 364-365, 259-275, doi : 10.3406/ outre.2009.4425.

Dunning J.H., Lundan S.M., 2008. Multinational enterprises and the global economy, Edward Elgar Publishing.

Edelblutte S., 2010. Paternalisme et territoires politiques dans la France de la seconde révolution industrielle : un regard rétrospectif sur les liens entre firmes et territoires communaux, Revue Géographique de l'Est, 50, 3-4, http:/ / rge.revues.org/3043.

Enjalric F., Ngoua Assoumou H.G., 1998. L'hévéaculture au Gabon, Plantations, Recherche, Développement, 5, 5, 325-332.

Enviropass, 2012. Étude d'impact environnemental et social en rapport avec l'aménagement d'une plantation industrielle d'hévéa sur le lot 4 dans les départements du Ntem et du Haut Ntem, Libreville.

Fitzherbert E.B., Struebig M.J., Morel A., Danielsen F., Brühl C.A., Donald P.F., Phalan B., 2008. How will oil palm expansion affect biodiversity?, Trends in Ecology $\mathcal{E}$ Evolution, 23, 538-545.

Gerber J.-F., 2011. Conflicts over industrial tree plantations in the South: who, how and why?, Global Environmental Change, 21, 165-176.

Gibbs H.K., Ruesch A.S., Achard F., Clayton M.K., Holmgren P., Ramankutty N., Foley J.A., 2010. Tropical forests were the primary sources of new agricultural land in the 1980s and 1990s, Proceedings of the National Academy of Sciences, $107,16732-16737$.

Hartemink A.E., 2005. Plantation agriculture in the tropics: environmental issues, Outlook on Agriculture, 34, 11-21.

Hirschman A.O., 1958. The strategy of economic development, New Haven, Yale University Press.
Koop K., Landel P.-A., Pecqueur B., 2010. Pourquoi croire au modèle du développement territorial au Maghreb? Une approche critique, EchoGéo, 13, http: / / echogeo.revues.org/ 12065.

Lambin E.F., Meyfroidt P., Rueda X., Blackman A., Börner J., Cerutti P.O., Dietsch T., Jungmann L., Lamarque P., Lister J., 2014. Effectiveness and synergies of policy instruments for land use governance in tropical regions, Global Environmental Change, 28, 129-140.

Latour B., 1999. Politiques de la nature: comment faire entrer les sciences en démocratie, Paris, La Découverte.

Loungou S., 1999. La frontière nord du Gabon: une brève étude de géographie politique, Espace populations sociétés, 3, 439-449.

Magnagna Nguema V., 2005. L'agriculture du Gabon: entre décolonisation et ajustements structurels (1960-2000), Paris, Karthala.

Magrin G., van Vliet G., 2006. Greffe pétrolière et dynamiques territoriales: l'exemple de l'on-shore tchadien, Afrique contemporaine, 216, 87-105.

Maisels F., Strindberg S., Blake S., Wittemyer G., Hart J., Williamson E.A., Aba'a R., Abitsi G., Ambahe R.D., Amsini F., 2014. Devastating decline of forest elephants in Central Africa, PLOS ONE, 8, 3, e59469.

McCarthy J., Zen Z., 2010. Regulating the oil palm boom: assessing the effectiveness of environmental governance approaches to agro-industrial pollution in Indonesia, Law $\mathcal{E}$ Policy, 32, 153-179.

N'Goulakia L.P., 2014. L'agriculture et les filières du café et du cacao : une ambition pour le Gabon, Géoéconomie, 70, 3, 8594.

Newton P., Agrawal A., Wollenberg L., 2013. Interventions for achieving sustainability in tropical forest and agricultural landscapes, CAPRi Working Paper, No. 110, Washington, D.C., International Food Policy Research Institute, http:/ / dx.doi.org/10.2499/CAPRiWP110.

Ovono Edzang N., 2008. Hévéaculture gabonaise et filières hévéicoles des pays de la CEMAC. Compétition et/ou complémentarité, Géo-Éco-Trop, 32, 61-74.

Paulin P., 2008. Les Pygmées Baka du Gabon: approche sociolinguistique, in Loiseau M., Abouzaïd M., Buson L., Bannour R., Comanescu F. et al. (Eds.), Autour des langues et du langage. Perspective pluridisciplinaire, Grenoble, Presses universitaires de Grenoble, 307-314.

Pourtier R., 1980. La crise de l'agriculture dans un État minier : le Gabon, Études rurales, 77, 39-62.

République gabonaise, 2012. Plan stratégique Gabon émergent. Vision 2025 et orientations stratégiques 2011-2016. Déclinaison en programmes et actions du projet de société de son Excellence Ali BongoOndimba, Président de la République: "l'Avenir en confiance ", Libreville.

Veltz P., 1993. D'une géographie des coûts à une géographie de l'organisation. Quelques thèses sur l'évolution des rapports entreprises/territoires, Revue économique, 44, 4, 671-684.

Vogel D., 2008. Private global business regulation, Annual Review Political Science, 11, 261-282. 